VII JORNADAS DE DIFUSIÓN DE LA INVESTIGACIÓN Y EXTENSIÓN - FCV-UNL

RESUMEN EXTENDIDO

\title{
PRIMER REPORTE DE LESIONES PARASITARIAS COMPATIBLES CON Oslerus osleri (COBBOLD, 1876) EN AGUARÁ GUAZÚ, Chrysocyon brachyurus (ILLIGER, 1811) DE LA PROVINCIA DE SANTA FE.
}

\author{
Torrents $\mathrm{J}^{1}$, Bagattin $\mathrm{L}^{2}$, Murillo $\mathrm{A}^{2}$, Sanchez $\mathrm{A}^{2}$, Marini $\mathrm{MR}^{2}$, \\ Sciabarrasi $A A^{1,3}$, Eberhardt $A T^{4}$, Marengo $\mathrm{RE}^{1}$ \\ ${ }^{1}$ Cátedra de Zoología, Diversidad y Ambiente FCV-UNL \\ ${ }^{2}$ Cátedra de Patología Veterinaria FCV- UNL \\ ${ }^{3}$ Servicio Veterinario Estación Zoológica Experimental Granja "La Esmeralda"- Dirección de Ecología \\ y Protección de Fauna- Ministerio de la Producción, Santa Fe \\ ${ }^{4}$ Laboratorio de Ecología de Enfermedades, Instituto de Ciencias Veterinarias del Litoral (UNL- \\ CONICET). \\ * Correspondencia: Torrents J. E-mail: torrentsjorgelina@gmail.com
}

Editado por: R. Sobrero, V. Matiller, C. Baravalle.

FIRST REPORT OF PARASITIC LESIONS COMPATIBLE WITH Oslerus osleri (COBBOLD, 1876) IN AGUARÁ GUAZÚ, Chrysocyon brachyurus (ILLIGER, 1811) FROM THE PROVINCE OF SANTA FE.

SUMMARY.

The necropsy of 5 specimens of $C$. brachyurus which were accidentally run over on different routes in the north-central province of Santa Fe. All of these had multiple whitish nodules with a cystic appearance between the distal third of the trachea and bronchi containing parasites arranged in the shape of a ball inside. Morphological identification of adult's nematodes and larvae eggs from females was performed, where it was possible to identify typical characters of the species $O$. osleris. Likewise, the lungs were increased in consistency, with petechial and multifocal ecchymosis and the presence of whitish nodules scattered throughout the parenchyma. Two of the canids had an increase in the size of the bronchial lymph nodes. The inspection of the respiratory sys tem together with the observation of lesions and the nematodes found inside with their corresponding measurements, are compatible with the species $O$. osleri, which would imply the first report of this parasite in wild canids from Argentina.

Palabras clave: encuesta, enfermedades caprinas, Chrysocyon brachyurus, Oslerus osleri, lesiones parasiticas, aguará guazú.

Keywords: Chrysocyon brachyurus, Oslerus osleri, parasitic lesions, maned wolf.

El aguará guazú, Chrysocyon brachyurus, es el mayor de los cánidos sudamericanos vivientes. Posee hábitos solitarios, nocturnos o crepusculares, este carnívoro puede considerarse oportunista, teniendo como base de su alimentación pequeñas presas como aves, mamíferos, reptiles y artrópodos, complementando su dieta con frutos silvestres. En Argentina se distribuye principalmente en las provincias de Santa Fe, Chaco y Corrientes (74\%). Otras provincias en las que se registra en menor número son Santiago del Estero, Córdoba, Formosa, Entre Ríos y Misiones (26\%). Se encuentra categorizado a nivel nacional como "Especie amenazada" (resolución SAyDS 1030/04), protegido por la Ley Nacional de Conservación de la Fauna 22.421/81, y a nivel internacional en el apéndice II de CITES por la UICN (Unión Mundial para la Naturaleza). Las causas que llevaron al aguará guazú al estado de conservación actual son la modificación de ambientes por la actividad 
agropecuaria, deforestación, construcción de rutas y captura debido a ser considerada una especie "rara". Todas estas causas, generan un efecto que puede favorecer la ocurrencia de enfermedades de diferentes etiologías. Entre ellas se encuentran las enfermedades parasitarias que naturalmente pueden estar en equilibrio con su hospedador. Este equilibrio, bajo ciertas situaciones de estrés de origen antrópico o natural puede verse afectado e influir en la dinámica poblacional de los hospedadores (Orozco et al., 2015).

Uno de los parásitos encontrados en caninos silvestres es Oslerus osleri (sin. Filaroides osleri). Este nematodo pequeño, pertenece a la superfamilia Metastrongyloidea, familia Filaroididae. Tiene como localización definitiva el tracto respiratorio y afecta tanto a cánidos silvestres como domésticos. Estos, se ubican en tráquea y bronquios, con predilección por la carina traqueal (Anderson, 2000; Taylor et al., 2015). este parásito se ha encontrado caninos silvestres de Europa, Norteamérica, Asia, África y Oceanía. en Sudamérica se ha encontrado en un perro en chile $y$ Argentina y en aguará guazú en Brasil.

El objetivo del presente trabajo es reportar por primera vez la presencia de O. osleri en aguará guazú en la Argentina y describir macroscópicamente las lesiones que produce en la pared de la tráquea y los bronquios.

Se realizó la necropsia de 5 individuos de $C$. brachyurus en la Facultad de Ciencias Veterinarias de la Universidad Nacional del Litoral. Los mismos fueron atropellados accidentalmente en diferentes rutas del centro-norte de la provincia de Santa Fe. En primer lugar, se inspeccionó externamente el aparato respiratorio completo. Luego se procedió a la apertura de las vías aéreas comenzando desde el primer anillo traqueal hasta bronquios. En la tráquea y bronquios de todos los ejemplares necropsiados se hallaron múltiples nódulos blanquecinos de aspecto quístico, que en su interior contenían parásitos dispuestos en forma de ovillo (Figura 1). Al extraerlos se pudo constatar que la morfología era compatible con la de nematodos. Esta maniobra resultó dificultosa, debido a que los nódulos se encontraban firmemente adheridos a la mucosa, de manera que al extraer los vermes los algunos se dañaron. Los parásitos obtenidos fueron fotografiados y medidos con el programa S-EYE (Real time measurement software for android). Se encontraron ejemplares de ambos sexos, machos con espículas
(Figura 2) y hembras con el útero con gran cantidad de huevos larvados en su interior (Figura 2). La longitud total promedio de machos fue $5 \mathrm{~mm}$, las hembras 12 $\mathrm{mm}$ y los huevos $95,098 \mu \mathrm{m} \times 59,783 \mu \mathrm{m}$. Asimismo, los pulmones se encontraban aumentados de consistencia, con petequias y equimosis multifocales y presencia de nódulos blanquecinos diseminados en todo el parénquima. En dos de los animales se presentaba un aumento del tamaño de los linfonodos bronquiales. La inspección del aparato respiratorio en conjunto con la observación de lesiones y los nematodos encontrados en el interior con sus correspondientes medidas, son compatibles con la especie $O$. osleri, lo cual implicaría el primer reporte de este parásito en cánidos silvestres de Argentina (Anderson, 2000; Taylor et al., 2015).

Oslerus osleri se transmite por regurgitación de larvas cuando la hembra adulta alimenta a sus cachorros, comportamiento característico de los cánidos silvestres como el aguará guazú, el cual implica un importante factor epidemiológico en la transmisión y perpetuación de este parásito. En Australia se realizaron los primeros estudios sobre esta especie, indicando que puede estar presente en una gran cantidad de cánidos silvestres. Se reportó una prevalencia mayor al 41,2\% (Dunsmore \& Spratt, 1979). Si bien, sólo se ha realizado la necropsia de 5 individuos de $C$. brachyurus hallados muertos accidentalmente, el hecho de provenir de distintas localidades y de haber encontrado el parásito y las lesiones en todos los ejemplares examinados, podría suponer que la prevalencia de este parásito en la población de $C$. brachyurus es alta, y es necesario continuar realizando estudios para poder determinarla.

Estudios previos han demostrado que la enfermedad producida por este parásito cursa de manera subclínica, en poblaciones de especies silvestres. Aunque en infecciones severas puede afectar la función respiratoria e inclusive llevar a su hospedador a la muerte. Este trabajo pretende resaltar la importancia de este hallazgo por primera vez en cánidos silvestres en la Argentina, contribuyendo al aumento de información de las enfermedades que pueden afectar la supervivencia del aguará guazú. Creemos que conocer los patógenos a los cuales esta especie es susceptible es un elemento de gran importancia para su conservación in situ y ex situ. 

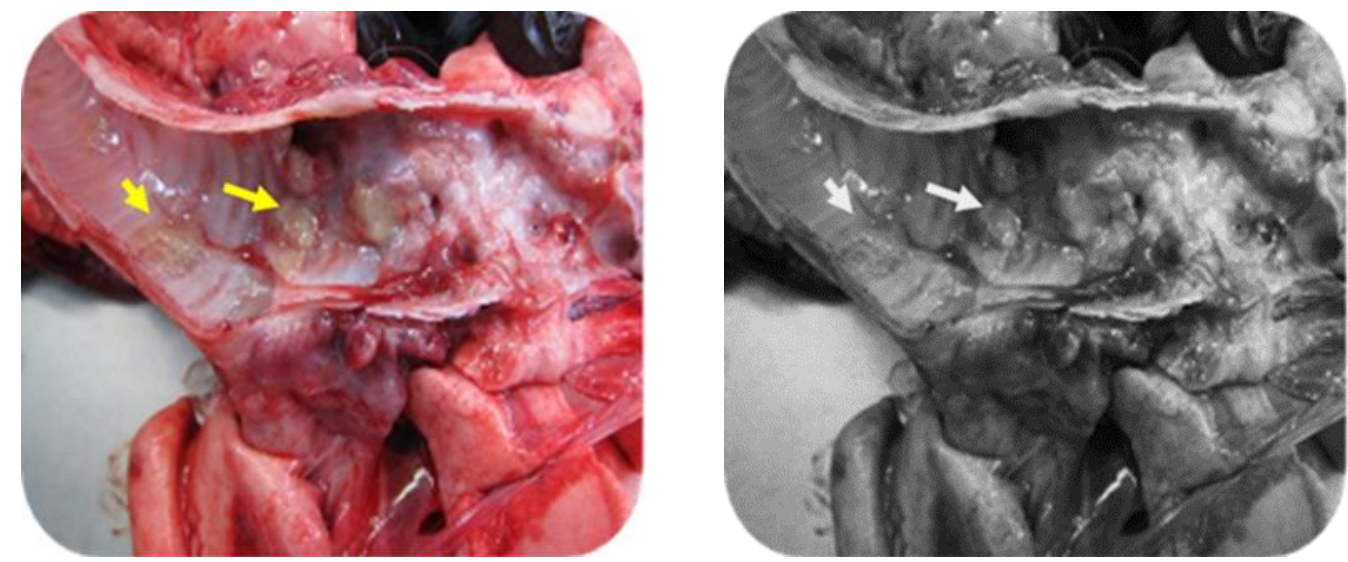

Figura 1. Versión color y blanco y negro. Nódulos blanquecinos hallados en tráquea a la altura de la bifurcación y bronquios
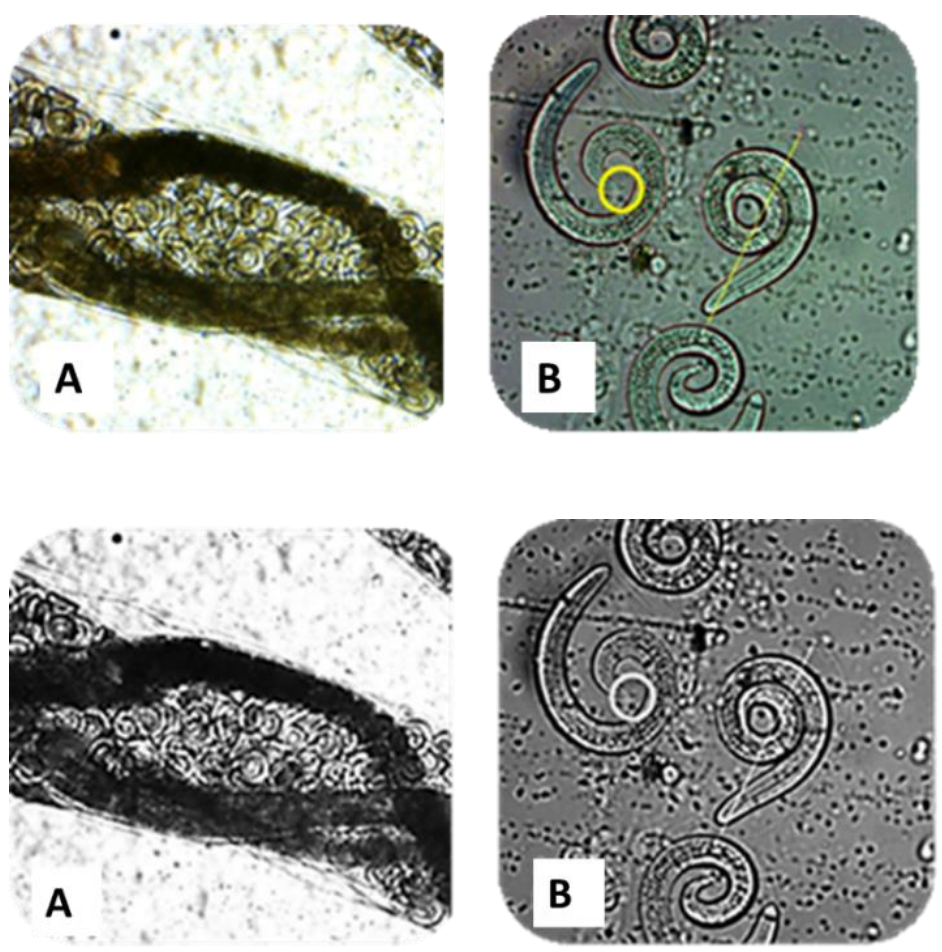

Figura 2. A: Versión color y blanco y negro. sección transversal de una hembra. Se observan huevos larvados dentro del útero. B: Huevos larvados, el extremo posterior de la larva tiene un apéndice en forma de S. Largo y ancho promedio de huevos: 95,098 $\mu \mathrm{m} \times 59,783 \mu \mathrm{m}$.

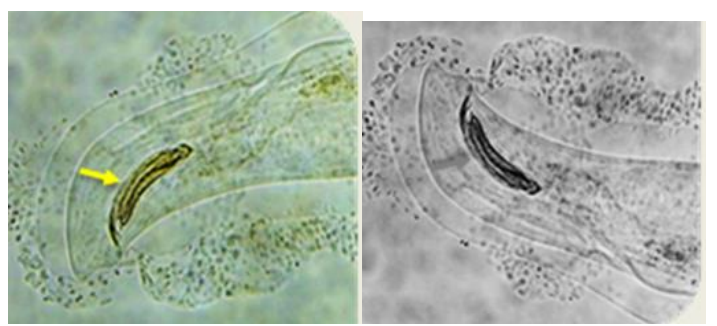

Figura 3. Versión blanco y negro. Porción posterior de un macho, la flecha amarilla señala las espículas. 


\section{Bibliografía}

Anderson RC. 2000. Nematode Parasites of Vertebrates, 2nd ed. CABI Publishing, New York, New York. 128-173 pp.

Dunsmore JD and Spratt DM. 1979. The life history of Filaroides osleri in wild and domestic canids in Australia. Vet. Parasit. 5: 275286.

Taylor MA, Coop RL, Wall RL. 2007. Veterinary parasitology, 3rd edn. Blackwell Publishing Ltd, UK 565 Orozco M, Ciccia P \& Soler L. 2015. El aguará guazú (Chrysocyon brachyurus) en la Argentina / Maned wolf (Chrysocyon brachyurus) in Argentina. $1^{\circ}$ edición. Fundación de Historia Natural Félix de Azara. Ciudad Autónoma de Buenos Aires, República Argentina.

Orozco M, Ciccia P \& Soler L. 2015. El aguará guazú (Chrysocyon brachyurus) en la Argentina / Maned wolf (Chrysocyon brachyurus) in Argentina. $1^{\circ}$ edición. Fundación de Historia Natural Félix de Azara. Ciudad Autónoma de Buenos Aires, República Argentina. 